\title{
Ultrasonographic Study of Femur Length in Fetuses of North Indian Women
}

\author{
Nishtha Singh $^{1}$, P. K. Sharma ${ }^{2}$, A. K. Singh ${ }^{1}$ \\ ${ }^{1}$ (Department of Anatomy, Moti Lal Nehru Medical College, Allahabad, India.) \\ ${ }^{2}$ (Department of Anatomy, Chhatrapati Shahuji Maharaj Medical University, Lucknow, India.)
}

\begin{abstract}
To identify and adequately manage cases of growth restriction and preterm delivery in a timely manner should be considered a public health priority, especially in developing countries. Fetal growth differs not only between individuals but also between populations. The purpose of this prospective, cross-sectional, observational study was to construct new reference charts and equations for fetal femur length in relation to menstrual age, in North Indian women with normal singleton pregnancies. 1081 pregnant women were scanned using real-time transabdominal ultrasonogram, 947 women fitted the inclusion criteria. Mean $\pm s d$ was computed and compared with available data of other population using t-test. Regression equation was calculated and the predicted femur length was compared with available data of other studies. The results suggest that fetal growth with respect to femur length may not be adequately assessed by using reference charts obtained from other populations. We have created new reference ranges for fetal femur length in North Indian population.
\end{abstract}

Key Words: Fetal femur length, Gestational age, Regression equation

\section{Introduction}

Gestational age, synonymous with menstrual age, is defined in weeks beginning from the first day of the last menstrual period (LMP) prior to conception. Accurate determination of gestational age is fundamental to obstetric care and is important in a variety of situations.

Measurement of various fetal body parts is known as fetal biometry. In common obstetric practice, the adequacy of fetal size is assessed by comparing the observed measurements of fetal anatomical parameters at a given gestational age with reference percentiles of fetal size calculated in populations of fetuses whose growth is assumed to be normal. The same reference charts can be used to estimate gestational age from observed fetal size. However, to ensure accurate diagnosis, the selection of the appropriate cross sectional reference charts is of great importance. Many charts and tables for assessing gestational age have been established and several standard charts have been fed into ultrasound machines for ready reference. Most of the commonly used charts of size by gestational age have been obtained from populations of fetuses in the USA or Europe and, as early as 1981. The concern has been raised that such charts might not be appropriate for use in populations of different ethnicity that could show different patterns of fetal growth. The use of locally developed charts means that the factors that may influence fetal biometry, including maternal age and nutritional status, maternal weight and size can be identified, facilitating accurate prediction of small for date and growth retarded foetuses. In this study we have tried to establish growth charts for fetal femur length for the North Indian population. All the fetal long bones can be adequately examined and measured by ultrasound; however, the femur is the largest of the long bones, least moveable, and easiest to image.

\section{Material and method}

Ultrasound examination of foetuses of North Indian women population was carried in a prospective, cross sectional, observational study to create reference ranges. Our study group consisted of North Indian women with singleton pregnancy, who were under follow up at Department of Obstetrics and Gynaecology and underwent their routine antenatal sonographic assessment in the department of Radiology of M. L. N. Medical College and associated hospitals, Allahabad.

Among 1081 pregnant women scanned, 947 women fitted the inclusion criteria. Ultrasound has no known harm to the pregnant females or their unborn foetuses. The pregnant females participated voluntarily after informed consent according to a protocol acknowledged by the medical college committee of medical research ethics.

All the women whether primigravida or multigravida were included in this study except for those who had -uncertain date of last menstrual period, any history of prolonged substance abuse (alcohol, smoking etc), Multiple pregnancies, Fetal malformations, Chronic maternal disease or medication that could affect the growth of the fetus. 
No fetuses were excluded on the basis of abnormal biometry or birth weight. The nutritional status of the pregnant female has not been taken into account, because in the context of North Indian females, excluding women with poor nutritional status or antenatal infections from the study population would create an 'artificially healthy' pregnancy cohort.

2.1 Data acquisition: For fetal biometric measurements, real-time transabdominal ultrasonogram was taken using a "Siemens G- 50" and "GE Logiq 400 pro" ultrasound machine equipped with a 3.5-MHz curvilinear transabdominal probe. The measurements were taken to the nearest millimetre.

2.2 Fetal biometrics: The technique originally described by O'Brien et al.[1] was used to align the transducer along longest axis of the femur. When the longest possible image of femur is visualized, the straight lateral surface is measured rather than medial surface which is bowed. Femur Length has been measured in a plane where the full femoral diaphysis is seen almost parallel to the transducer and the measurement is made from one end of the diaphysis to another (Fig. 1). The fetal femur can be identified in utero using real time sonography by $10^{\text {th }}$ menstrual week. In the third trimester, particular care was taken not to include the epiphysis in the measurement. The distal femoral epiphyses are visible after 32 weeks. The proximal tibial epiphysis becomes visible at around 35 weeks.

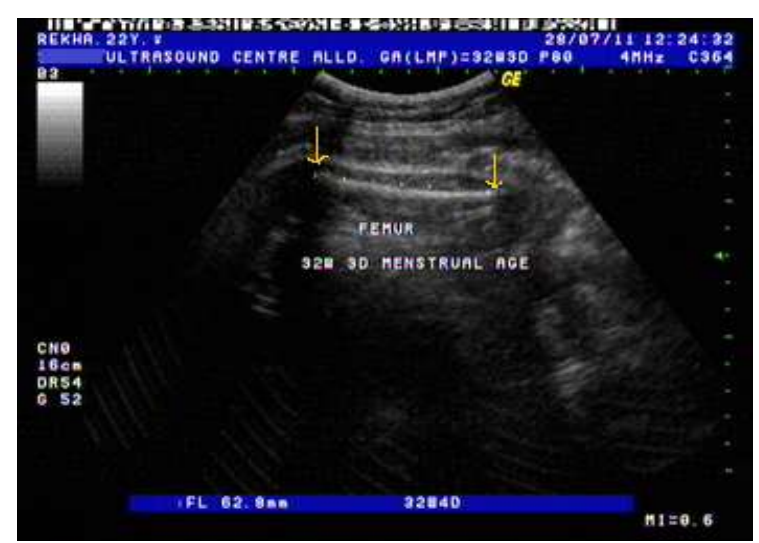

Fig. 1 : The distance between the 2 arrows is measured as femur length.

2.3 Statistical analysis: The data were entered and analyzed in a computer, using MS Excel 07 and Statistical Package for Social Sciences (SPSS) version 10.0. The descriptive statistics (mean and standard deviation) were performed for the measurements of femur lengths at weekly intervals. Each interval was centred on a week, so that from 9 weeks and 4 days up to 10 weeks and 3 days has been considered as $10^{\text {th }}$ week. A regression analysis was done examining linear $(\mathrm{FL}=\mathrm{a}+\mathrm{b} \times \mathrm{GA})$, cubic $\left(\mathrm{FL}=\mathrm{a}+\mathrm{b} \times \mathrm{GA}+\mathrm{c} \times \mathrm{GA}^{2}\right)$ and quadric $(\mathrm{FL}=\mathrm{a}$ $\left.+b \times G A+c \times G A^{2}+d \times G A^{3}\right)$ models for the association with gestational age. The regression equations used to generate the charts and tables for femur length were thus derived. The best fit model was decided by calculating Pearson correlation coefficient ( $\mathrm{r} 2$ ) to compare the strength of the association for the regression model in each case.

Assessment of the quality of mathematical relationship and analysis of difference between 2 groups was performed by using unpaired t-test.

\section{Observation}

Among 1081 pregnant women scanned, 947 women fitted the inclusion criteria. A total of 134 women were excluded for the following reasons: no information on the date of their last menstrual period ( $n=61)$, femur too small to be visible $(n=67)$, multiple foetuses $(n=2)$ and fetal malformation $(n=3)$. Among 947 females $57.23 \%$ belonged to urban area and the rest $42.77 \%$ were from rural area. The study group consisted mainly of young females. Their mean age was 25.34yrs (range 18 years to 37 years). In the week vice distribution, the number of females was more and almost equally distributed from 12 to 39 weeks $(2-6 \%)$. It was less in $10^{\text {th }}$, $11^{\text {th }}, 40^{\text {th }}$ and 42 weeks $(0.32,0.21,1.16$ and $0.53 \%$ respectively). The presentation of the foetuses was predominantly cephalic $(70.33 \%)$. Breech presentation was seen in $17.53 \%$ of foetuses. Transverse presentation was the least (1.27\%). Early stages of pregnancy showed unstable transverse or unstable breech lie (10.88\%)

Raw data was fitted in Linear, square and cubic equations and tables for femur length were generated. The best fit model for estimating femur length from gestational age, in our study was square regression equation. The formula and the correlation coefficient $\left(r^{2}\right)$ was as follows-

Square regression equation: 
$\mathrm{FL}=-42.3823+4.58067 \times \mathrm{GA}-0.0404791 \times \mathrm{GA}^{2}$

$\left(r^{2}=92.05\right)$

Here FL is femur length in millimetres with 2 decimal places and GA is menstrual gestational age (gestational age calculated by last menstrual period) in exact weeks i.e. 10 weeks 1 day is 10.14 weeks, 10 week 2 day is 10.29 weeks and so on. The regression analyses showed that femur length correlated significantly with gestational age at $99 \%$ confidence limit ( $P$-value in ANOVA table is less than 0.01$)$. The predicted femur lengths at weekly points in gestation are indicated in Fig. 2.

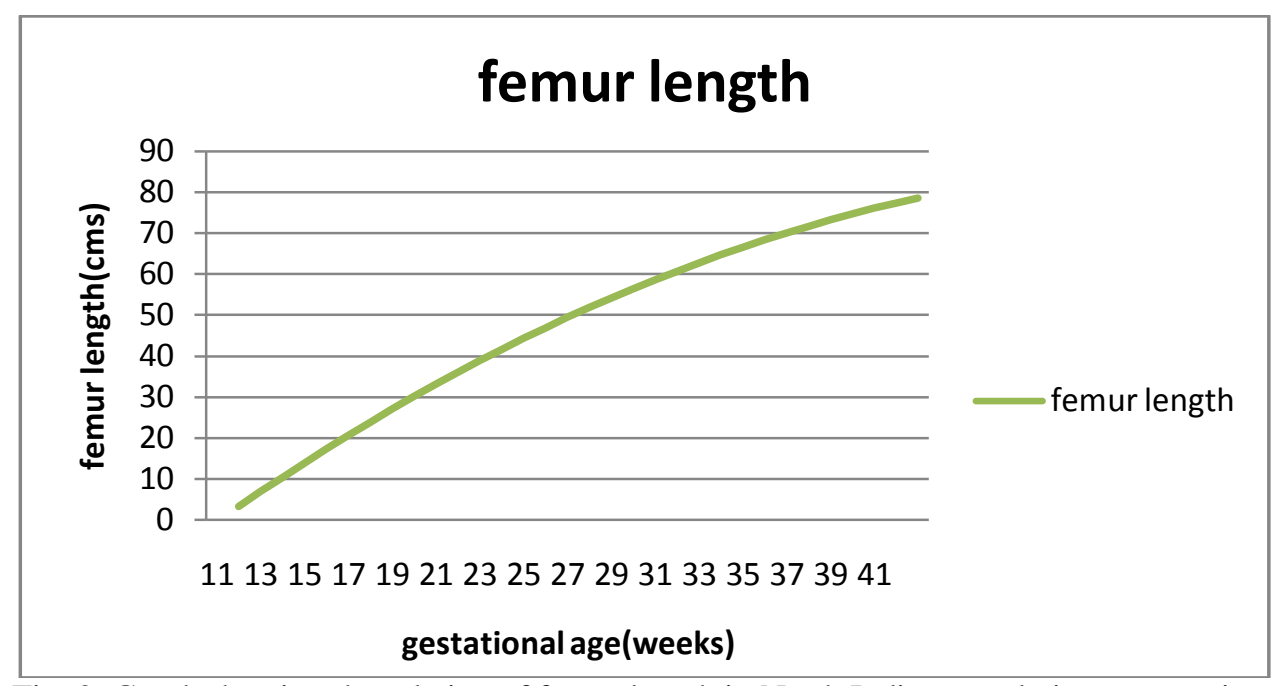

Fig. 2: Graph showing the relation of femur length in North Indian population to gestational age.

\section{Discussion}

In this cross-sectional study we have tried to establish normal ranges for femur length for the North Indian population. Each woman contributed to one set of measurements, taken during a routine ultrasound examination.

The regression analysis tables of various studies shows that the predicted femur length in the present study is almost equal to the predicted femur length in the study done by Hadlock et. al. [2] in 338 normal foetuses.

Much difference is seen in the predicted value of femur length in the present study and the study carried by Jeanty et. al. [3] in 450 pregnancies. The difference in the predicted value of femur length gradually increases by $2-11 \mathrm{mms}$ from 12 to 40 weeks.

The comparison of predicted femur length of our study population (North Indian) with those of the study by T. N. Leung et. al. [4] in a study involving 709 pregnancies in China shows that femur lengths of Chinese foetuses are 1-4mms smaller than North Indian foetuses throughout 12 weeks to term.

Femur length predicted by Pam Loughna et. al. [5] in United Kingdom is about 1-2 mms less than the predicted femur length of our study in most the weeks. There is almost no difference in the predicted femur lengths of our study and the study by Tamy Shohat et. al. [6], involving 1143 pregnancies in Israel.

To find out whether there is any statistical difference between the mean and standard deviation of any of the two studies, 't' test has been done for each week. If the $P$ value is less than or equal to 0.05 , the difference is considered statistically significant.

In the study involving 358 subjects in Pakistan by Shahida Zaidi et. al. [7], the 't' test shows significant value only in $16^{\text {th }}$ week. This means that the difference in the means which shows that femur length in Pakistan foetuses is greater than ours is not significant. This may be because majority (68\%) of their cohort comprised of second and third generation immigrants from north India.

Comparison of the femur length obtained in the present study with those of study carried in Northeastern Thailand by Piyamas Saksiriwuttho et. al. [8] involving 635 pregnancies showed that the femur lengths are almost equal up to 20 weeks. There after there was a steady difference of 2-4 mms up to 41 weeks. The' $t$ ' test shows a significant $P$ value in most of the weeks, which implies that the femur lengths of Thai foetuses are smaller than the North Indian foetuses. The differences could be attributed to racial origin, that the Thai foetuses being smaller than ours.

On comparing the mean femur length with those of the study of R. J. M. Snijders et. al. [9] carried in London; the ' $t$ ' test shows significant difference only in initial weeks, the femur length of their foetuses being on higher side. But in later weeks there is no significant difference between the two means. 


\section{Conclusion}

To assess the gestational age with the help of ultrasound, we have tried to establish normal ranges of fetal growth for femur length measurements from 10 weeks of gestation onwards.

Regression equation for derivation of tables of femur length in North Indian population is $\mathrm{FL}=-42.3823+$ $4.58067 \times \mathrm{GA}-0.040791 \times \mathrm{GA}^{2}$ ( $\mathrm{FL}=$ femur length, $\mathrm{GA}=$ gestational age in weeks), and tables derived from this equation can be used as reference charts.

Comparison of mean femur length of North Indian fetuses with the studies on femur length of fetuses in United Kingdom, Pakistan and Israel showed not much significant differences while the femur length of the Thai and Chinese foetuses was found to be smaller than North Indian fetuses. The differences could be attributed to racial factors, dietary factors etc.

There was no difference in the predicted femur length of fetuses in our study in comparison to the study done by Hadlock et. al. Hence table of femur length for dating pregnancy proposed by Hadlock et. al. as well as table derived from our study can be used for estimating gestational age in the fetuses of North Indian population.

Femur growth is relatively preserved in Indian foetuses.The lower newborn length at birth in North Indian population than in the United Kingdom neonates suggests a greater relative deficit in components of fetal length other than femur length: for example, the spine or head.

\section{References}

[1] G. D. O'Brien and J. T. Queenan, Growth of the ultrasound fetal femur length during normal pregnancy. Part I, American Journal of Obstetrics and Gynecology, 141(7), 1981, 833-837.

[2] F. P. Hadlock, R. B. Harrist, R. L. Deter and S. K. Park, Fetal femur length as a predictor of menstrual age: sonographically measured, American Journal of Roentgenology, 138 (5), 1982, 875-878.

[3] P. Jeanty, C. Kirkpatrick, M. Dramaix-Wilmet, J. Struyven, Ultrasonic evaluation of fetal limb growth; Radiology, 140(1), 1981, 1658.

[4] T. N. Leung, M. W. Pang,S. S. Daljit, T.Y. Leung, C. F. Poon, S. M. Wong, T. K. Lau, Fetal biometry in ethnic Chinese: biparietal diameter, head circumference, abdominal circumference and femur length. Ultrasound Obstet Gynecol, 31, $2008,321-327$.

[5] Pam Loughna, Lyn Chitty, Tony Evans, Trish Chudleigh. Fetal size and dating: charts recommended for clinical obstetric practice. Ultrasound, 17(3),2009, 161-167

[6] Tamy Shohat, Orly Romano-Zelekha. Ultrasonographic measurements of fetal femur length and biparietal diameter in an Israeli population. IMAJ, 3, 2001,166-168.

[7] Shahida Zaidi, Khalid Shehzad, Amir Omair. Sonographic foetal measurements in a cohort of population of Karachi, Pakistan. Journal of Pak Med Assoc, 59 (4), 2009, 246-249.

[8] Piyamas Saksiriwuttho, Thawalwong Ratanasiri, Ratana Komwilaisak Fetal Biometry Charts for Normal Pregnant Women in Northeastern Thailand. J Med Assoc Thailand, 90 (10), 2007, 1963-1969

[9] R. J. M. Snijders and K. H. Nicolaides, Fetal biometry at 14-40 weeks gestation. Ultrasound obstet. Gynecol.,4, 1994, 34-48. 